\title{
Asian gastric cancer patients show superior survival: the experiences of a single Australian center
}

\author{
Yufei Chen · Jan Willem Haveman • \\ Christos Apostolou • David K. Chang • \\ Neil D. Merrett
}

Received: 6 September 2013/Accepted: 23 April 2014/Published online: 25 May 2014

(C) The International Gastric Cancer Association and The Japanese Gastric Cancer Association 2014

\begin{abstract}
Background Survival after curative gastrectomy for gastric cancer varies depending on region. The 5-year survival rates in Western trials reach 36-47\% compared with 40-60\% in Japanese studies. We analyzed the outcomes of Asian and non-Asian patients at a single Australian institution.

Methods We analyzed a prospectively kept database of patients following gastric resection between 1994 and 2010 at a tertiary Australian hospital. Overall survival was the primary endpoint.

Results A total of 160 patients underwent a R0 gastrectomy with curative intent, of whom $26(16 \%)$ were of Asian descent. Asian patients had a significantly younger age at diagnosis $(60 \pm 16$ vs. $70 \pm 11, p<0.05)$ and longer overall survival (log-rank $p=0.018$ ). Poor prognostic factors common to both groups included increased tumor length, higher T-score, higher LN ratio, poor tumor differentiation, and the presence of perineural or perivascular invasion. Multivariate analysis showed that nonAsian patients, higher T-score, higher $\mathrm{N}$-score, and perivascular involvement were all independent predictors of poorer outcome.
\end{abstract}

Y. Chen · J. W. Haveman - C. Apostolou .

D. K. Chang · N. D. Merrett

Department of Upper GI Surgery, Bankstown Hospital,

Bankstown, Sydney, NSW, Australia

Y. Chen $(\bowtie)$

10 Oakes Avenue, Eastwood, NSW 2122, Australia

e-mail: yufeichen@gmail.com

N. D. Merrett

Discipline of Surgery, University of Western Sydney, Sydney,

NSW, Australia
Conclusions This study shows superior overall survival in Asian patients despite similar clinicopathological and treatment data. The younger age at diagnosis in Asian patients may suggest a different disease process between ethnicities. Targeted therapies based on population-specific tumor biology may potentially be beneficial.

Keywords Stomach neoplasms - Gastrectomy · Ethnicity · Asians

\section{Introduction}

Gastric cancer remains a significant cause of morbidity and mortality, and is the second leading cause of cancer-related death worldwide [1]. The incidence and mortality rates, particularly in developed countries, have decreased steadily over the past few decades, which has been attributed to factors such as dietary improvements and earlier detection and management of Helicobacter pylori infection [2].

Gastric cancer within Australia remains a deadly disease, with a 5 -year relative survival of less than $25 \%$. In 2007, there were 1,897 new diagnoses of gastric cancer with 1,129 deaths [3]. It is the 11th most common cancer in Australia. with an age-standardized incidence of 8.7 per 100,000 and the 8th most common cause of cancer death, with age-standardized death rate of 5.0 per 100,000 [3].

Throughout the literature, marked geographic variations in the incidence of gastric cancer have been shown with populations in Eastern Asia, Central and South America, and Eastern Europe at significantly higher risk [4]. Overall 5 -year stage-specific survival has been repeatedly demonstrated to be markedly superior in studies from Japan and Korea when compared with large Western trials. This difference has been attributed to a variety of factors, 
ranging from more extended lymphadenectomy and stage migration to differences in tumor biology [5].

Australia has strong geographic and economic links with Asia and is notable for a large Asian migrant population, the majority of whom are overseas born and have come to Australia since the 1970s after the removal of restrictive immigration policies. In our study, we investigated the characteristics of Asian and non-Asian gastric cancer patients and their outcomes at a single Australian institution.

\section{Methods}

We analyzed a prospectively kept database of 191 patients following gastric resection for gastric adenocarcinoma between 1994 and 2010 at a tertiary Australian hospital. Recorded data included patient demographics (age, gender, and ethnicity), use of neoadjuvant or adjuvant chemotherapy and/or radiotherapy, pathological reports, operation details, perioperative morbidity, 30-day mortality, and survival status. All tumors were staged according to the American Joint Committee on Cancer (AJCC) 7th edition manual. Tumor differentiation was grouped into wellmoderate and poorly differentiated. Depth of tumor invasion was grouped into T1-2 and T3-4. Patients whose ethnicity was from East or Southeast Asian descent were considered Asian for the purposes of this analysis, with all notably born overseas: these included patients from China, Vietnam, Thailand, and Indonesia.

All patients were evaluated by a senior surgeon with a standardized investigation and treatment algorithm that included routine computed tomography (CT) scanning of all patients, routine endoscopic ultrasound (EUS) from 2004 onward, and positron emission tomography (PET)-CT scanning from 2007. All patients were evaluated in a comprehensive multidisciplinary treatment group to obtain consensus treatment decisions. Operations were performed by a small group of experienced surgeons who were trained in the same dissection and reconstructive methods. All patients received either a radical subtotal (RST) or a radical total (RTG) gastrectomy with a D1+ lymphadenectomy or D2 lymphadenectomy in selected patients. Our standard adjuvant therapy was an ECF (epirubicin, cisplatin, fluorouracil)- or ECX (capecitabine)-based regimen for 6 months.

Overall survival was the primary endpoint and was calculated from date of diagnosis of gastric cancer until date of death or date of last follow-up. The follow-up cutoff date was August 31, 2011. Patients who remained alive by the cutoff date were censored at that date. Median survival was calculated by Kaplan-Meier analysis, and survival curves were compared using the log-rank test.
All patient data were recorded in Microsoft Excel for Windows 2010 and analyzed using SPSS (version 20; IBM SPSS). Comparison of clinical and pathological data used the Mann-Whitney $U$ test for continuous variables and the chi-square test for categorical variables. Factors significant by univariate analysis were used in a multivariate Cox regression model to calculate hazard ratios (HR) and $95 \%$ confidence intervals. A $p$ value $<0.05$ was considered statistically significant.

Ethics approval was obtained from Sydney South West Area Health Service (SSWAHS) Human Research Ethics Committee (HREC) Western Zone.

\section{Results}

A total of 160 patients underwent a gastrectomy with curative intent; 13 patients had a R1 resection and 18 patients had a palliative procedure and were thus excluded. Of the included patients, 26 (16\%) were identified to be of Asian ethnicity. The demographics of our patient cohort are given in Table 1. Overall follow-up duration was to death or more than 36 months in $77 \%$ of Asian patients and $80 \%$ of non-Asian patients. Follow-up to 5 years was seen in 73 and $67 \%$ of patients in each group, respectively (Fig. 1). Seven (4\%) patients were not seen in the preceding 12 months of the study end date and were considered lost to follow-up. These patients had a median followup duration of 36.7 months (range, 7.9-89 months) and were included in the final analysis censored at the date of last follow-up.

The average age at diagnosis of Asian patients with gastric cancer was 60 years (range, 31-82 years) compared with 70 years (range, 35-91 years) for non-Asians $(p=0.01)$. The male-to-female ratio was similar between the two groups, both with a male preponderance.

There was no significant difference between the two groups in terms of surgical or pathological data, which included tumor length, operation type, number of lymph nodes examined and involved, lymph node ratio, and AJCC stage. Also, there was no significant difference in tumor differentiation and presence of perineural or perivascular invasion.

More Asian patients underwent adjuvant chemotherapy (50 vs. $28 \%$ ), with no difference in the use of neoadjuvant therapy. Perioperative morbidity was similar between the Asian and non-Asian groups (27 vs. $31 \%)$, as was the 30-day mortality (4 vs. $6 \%$ ). The median length of hospital stay was 12 days for Asian patients and 14 days for nonAsian patients $(p=0.103)$.

Median overall survival was superior in Asian patients when compared with non-Asian patients. The 3-year survival in Asian and non-Asian patients was 70 and $47 \%$, 
Table 1 Patient demographics and tumor characteristics: comparison between Asian and non-Asian patients

\begin{tabular}{|c|c|c|c|c|c|}
\hline & \multirow[t]{2}{*}{ Asians $(n=26)$} & \multirow[t]{2}{*}{ Non-Asians $(n=134)$} & \multirow[t]{2}{*}{$p$ value } & \multicolumn{2}{|c|}{ Univariate analysis } \\
\hline & & & & Hazard ratio & $p$ value \\
\hline \multicolumn{6}{|l|}{ Ethnicity } \\
\hline Asian & & & & 1 & $p=0.02$ \\
\hline Non-Asian & & & & $2.6(1.1-6.1)$ & \\
\hline \multicolumn{6}{|l|}{ Age } \\
\hline Average (range) & $60(31-82)$ & $70(35-91)$ & $p=0.01$ & $1.0(1.0-1.0)$ & $p=0.06$ \\
\hline \multicolumn{6}{|l|}{ Gender } \\
\hline Female & $8(31 \%)$ & $36(27 \%)$ & $p=0.81$ & 1 & \\
\hline Male & $18(69 \%)$ & $98(73 \%)$ & & $1.2(0.7-2.1)$ & $p=0.56$ \\
\hline \multicolumn{6}{|l|}{ Tumour length } \\
\hline$<3 \mathrm{~cm}$ & $16(62 \%)$ & $70(52 \%)$ & $p=0.40$ & 1 & \\
\hline$>3 \mathrm{~cm}$ & $10(38 \%)$ & $64(48 \%)$ & & $2.1(1.3-3.4)$ & $p<0.01$ \\
\hline \multicolumn{6}{|l|}{ Operation type } \\
\hline RST & $12(46 \%)$ & $56(42 \%)$ & $p=0.83$ & 1 & \\
\hline RTG & $14(54 \%)$ & $78(58 \%)$ & & $1.6(1.0-2.6)$ & $p=0.07$ \\
\hline \multicolumn{6}{|l|}{ Splenectomy } \\
\hline No & $20(77 \%)$ & $108(81 \%)$ & $p=0.79$ & 1 & \\
\hline Yes & $6(23 \%)$ & $26(19 \%)$ & & $1.7(1.0-2.9)$ & $p=0.05$ \\
\hline \multicolumn{6}{|l|}{ Pancreatectomy } \\
\hline No & $20(77 \%)$ & $112(84 \%)$ & $p=0.41$ & 1 & \\
\hline Yes & $6(23 \%)$ & $22(16 \%)$ & & $1.8(1.1-3.1)$ & $p=0.03$ \\
\hline \multicolumn{6}{|l|}{ Colectomy } \\
\hline No & $25(96 \%)$ & $120(90 \%)$ & $p=0.47$ & 1 & \\
\hline Yes & $1(4 \%)$ & $14(10 \%)$ & & $3.4(1.8-6.4)$ & $p<0.01$ \\
\hline \multicolumn{6}{|l|}{ T-score } \\
\hline $\mathrm{T} 1-2$ & $12(46 \%)$ & $73(54 \%)$ & $p=0.52$ & 1 & \\
\hline $\mathrm{T} 3-4$ & $14(54 \%)$ & $61(46 \%)$ & & $2.7(1.7-4.4)$ & $p<0.01$ \\
\hline \multicolumn{6}{|l|}{$\mathrm{LN}$ involved } \\
\hline No & $10(38 \%)$ & $52(39 \%)$ & $p=1.00$ & 1 & \\
\hline Yes & $16(62 \%)$ & $82(61 \%)$ & & $3.6(2.0-6.4)$ & $p<0.01$ \\
\hline \multicolumn{6}{|l|}{ LN examined } \\
\hline Median (range) & $20(8-45)$ & $21(1-59)$ & $p=0.39$ & $1.0(1.0-1.0)$ & $p=0.30$ \\
\hline \multicolumn{6}{|l|}{$>15 \mathrm{LN}$ examined } \\
\hline Yes & $21(81 \%)$ & $110(82 \%)$ & $p=1.00$ & 1 & \\
\hline No & $5(19 \%)$ & $24(18 \%)$ & & $1.0(0.5-1.8)$ & $p=0.96$ \\
\hline \multicolumn{6}{|l|}{$\mathrm{LN}$ ratio } \\
\hline 0 & $10(38 \%)$ & $52(39 \%)$ & $p=0.86$ & 1 & \\
\hline$<20 \%$ & $6(23 \%)$ & $36(27 \%)$ & & $3.2(1.7-6.3)$ & $p<0.01$ \\
\hline $20-40 \%$ & $6(23 \%)$ & $22(16 \%)$ & & $2.8(1.3-5.9)$ & $p=0.07$ \\
\hline$>40 \%$ & $4(15 \%)$ & $24(18 \%)$ & & $5.8(2.9-11.9)$ & $p<0.01$ \\
\hline \multicolumn{6}{|l|}{ AJCC stage } \\
\hline 1 & $8(31 \%)$ & $45(36 \%)$ & $p=0.92$ & 1 & \\
\hline 2 & $7(27 \%)$ & $38(28 \%)$ & & $2.6(1.3-5.4)$ & $p<0.01$ \\
\hline 3 & $11(42 \%)$ & $51(38 \%)$ & & $4.7(2.4-9.3)$ & $p<0.01$ \\
\hline \multicolumn{6}{|l|}{ Differentiation } \\
\hline Well-moderate & $8(31 \%)$ & $63(47 \%)$ & $p=0.14$ & 1 & \\
\hline Poor & $18(69 \%)$ & $71(53 \%)$ & & $2.1(1.3-3.4)$ & $p<0.01$ \\
\hline Perineural & & & & & \\
\hline
\end{tabular}


Table 1 continued

\begin{tabular}{|c|c|c|c|c|c|}
\hline & \multirow[t]{2}{*}{ Asians $(n=26)$} & \multirow[t]{2}{*}{ Non-Asians $(n=134)$} & \multirow[t]{2}{*}{$p$ value } & \multicolumn{2}{|c|}{ Univariate analysis } \\
\hline & & & & Hazard ratio & $p$ value \\
\hline No & $15(58 \%)$ & $92(69 \%)$ & $p=0.36$ & 1 & \\
\hline Yes & $11(42 \%)$ & $42(31 \%)$ & & $3.3(2.1-5.4)$ & $p<0.01$ \\
\hline \multicolumn{6}{|l|}{ Perivascular } \\
\hline No & $18(69 \%)$ & $88(66 \%)$ & $p=0.82$ & 1 & \\
\hline Yes & $8(31 \%)$ & $46(34 \%)$ & & $3.7(2.3-6.0)$ & $p<0.01$ \\
\hline \multicolumn{6}{|l|}{ Neoadjuvant therapy } \\
\hline No & $26(100 \%)$ & $122(91 \%)$ & $p=0.22$ & 1 & \\
\hline Yes & 0 & $12(9 \%)$ & & $2.3(1.1-4.8)$ & $p=\mathbf{0 . 0 3}$ \\
\hline \multicolumn{6}{|l|}{ Adjuvant therapy } \\
\hline No & $13(50 \%)$ & $97(72 \%)$ & $p=0.04$ & 1 & \\
\hline Yes & $13(50 \%)$ & $37(28 \%)$ & & $0.7(0.4-1.2)$ & $p=0.20$ \\
\hline \multicolumn{6}{|l|}{ Morbidity } \\
\hline No & $19(73 \%)$ & $93(69 \%)$ & $p=0.82$ & & \\
\hline Yes & $7(27 \%)$ & $41(31 \%)$ & & & \\
\hline \multicolumn{6}{|l|}{ 30-day mortality } \\
\hline Number (\%) & $1(4 \%)$ & $8(6 \%)$ & $p=1.00$ & & \\
\hline \multicolumn{6}{|l|}{ Length of stay (days) } \\
\hline Median (range) & $12(7-51)$ & $14(0-136)$ & $p=0.14$ & & \\
\hline \multicolumn{6}{|l|}{ Duration of follow-up } \\
\hline Until death or $>3$ years & $20(77 \%)$ & $107(80 \%)$ & $p=0.79$ & & \\
\hline Until death or $>5$ years & $19(73 \%)$ & $90(67 \%)$ & $p=0.65$ & & \\
\hline 3-year overall survival & $70 \%(n=20)$ & $47 \%(n=107)$ & $p=0.09$ & & \\
\hline 5-year overall survival & $68 \%(n=19)$ & $31 \%(n=90)$ & $p<0.01$ & & \\
\hline
\end{tabular}

Bold values indicate significant variables on univariate analysis

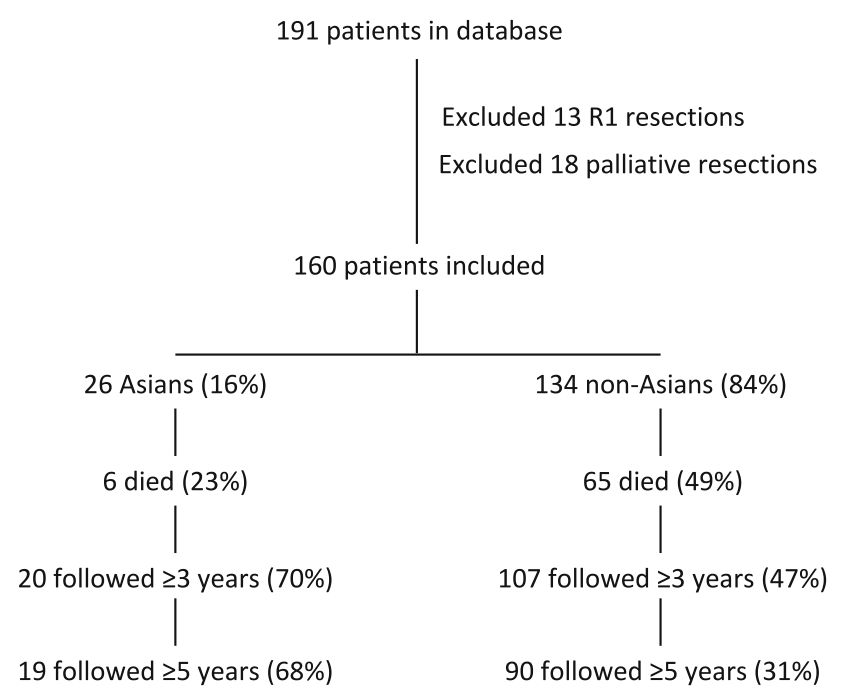

Fig. 1 Patient flow

respectively; at 5 years, this was 68 and $31 \%$ (Fig. 2). These results were shown to be statistically significant on log-rank comparison $(p=0.02)$.
Univariate analysis (Table 1) revealed that non-Asian ethnicity and increasing age were demographic features associated with worse outcomes. Having an associated splenectomy, pancreatectomy, or colectomy, increased tumor size, T-score, lymph node involvement, increased lymph node ratio, and higher AJCC stage were significant poor predictive factors. Negative pathological features included poor differentiation and evidence of perineural or perivascular invasion. Patients who underwent neoadjuvant therapy or had postoperative morbidity had significantly poorer overall survival.

On multivariate analysis (Table 2), when appropriate significant factors were considered together, only nonAsian ethnicity, higher $\mathrm{T}$-score, higher $\mathrm{N}$ stage, and perivascular invasion were independent predictors of poor outcome.

\section{Discussion}

To our knowledge, our study is the largest single-center study of gastric cancer patients conducted in Australia. The 


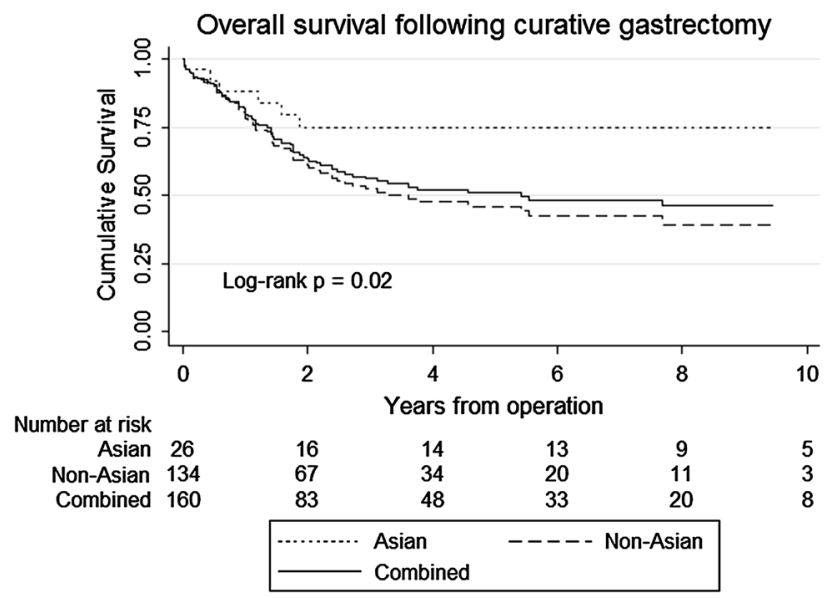

Fig. 2 Overall survival following curative gastrectomy

Table 2 Multivariate analysis: independent predictors of outcome

\begin{tabular}{llll}
\hline Variable & & Hazard ratio & $p$ value \\
\hline Ethnicity & Asian & 1 & \\
T-score & Non-Asian & $3.27(1.39-7.71)$ & $p=0.01$ \\
& T1-2 & 1 & \\
N-score & T3-4 & $1.69(1.00-2.84)$ & $p=0.05$ \\
& N0 & 1 & \\
& N1 & $2.08(0.99-4.37)$ & $p=0.05$ \\
& N2 & $2.38(1.15-4.93)$ & \\
Perivascular invasion & N3 & $2.70(1.30-5.59)$ & \\
& No & 1 & \\
& Yes & $2.43(1.46-4.07)$ & $p<0.01$ \\
\hline
\end{tabular}

relative homogeneity of the study in which all operations were performed by a small cohort of surgeons using identical staging and treatment algorithms and operative techniques minimizes the effect of individual surgical technique and expertise as a confounder in outcomes. It also reduces the variability in pathological analysis and staging accuracy that can often affect multi-institutional trials and analyses of cancer registries.

Our study found that Asian patients were significantly younger at diagnosis of gastric cancer when compared with non-Asian patients. There was no statistical difference in tumor stage or lymph node involvement at diagnosis. This trend was also identified in large epidemiological studies conducted in both Eastern and Western countries [5-7]. These data suggest that Asian patients are younger at diagnosis, possibly for reasons of genetic or environmental risk factors rather than earlier detection in itself.

This younger age at diagnosis may partly explain the improved survival seen in the Asian population. Other potential cofounders that were not assessed include the tendency for Asian patients to have a lower body mass index (BMI) than their Western counterparts [8]. Patients with fewer comorbidities and lower BMI have been shown to have fewer complications following gastrectomy and are less likely to have disease recurrence [9, 10]. However, there has been no correlation with lower BMI and increased survival [10].

Our study also found a significantly higher rate of use of adjuvant chemotherapy in the Asian group. Given that the tumor factors are similar between the two groups, this difference is likely accounted for by a more appropriate patient profile in Asian patient for chemotherapy selection, indirectly suggested by the younger age at diagnosis. However, this did not prove to be a significant factor accounting for improved survival.

Certain limitations of this retrospective analysis should be recognized. As a single-center study, there are undoubtedly implications with obtaining a larger sample sizes when compared to larger multicenter trials. Our study was underpowered to compare differences amongst Asian ethnicities and consequently grouped all Asian ethnicities together. Multiple epidemiological studies have shown that among Asian patients, those of Japanese and Korean ethnicity have the best gastric cancer survival rates $[5,11]$; this tendency persists even in migrants in Western countries [12]. Interestingly, our study did not include anyone from Japanese or Korean ethnicity, which may have underestimated the overall survival benefit of the Asian cohort.

\section{Conclusion}

Differences in the natural history of gastric cancer seen in Asians and non-Asians are reflected in the Australian population. Asian patients are more likely to be diagnosed at a younger age and show improved overall survival despite a similar extent of disease at diagnosis. This difference in epidemiology may represent variations in tumor biology, although further studies with larger numbers may be needed to evaluate this factor. Future targeted therapies based on population-specific tumor biology may potentially be beneficial.

Acknowledgments There are no sources of funding to declare.

Conflict of interest There are no conflicts of interest to declare.

\section{References}

1. World Health Organization. Cancer: Fact Sheet No. 297. February 2012. Available at: http://www.who.int/mediacentre/fact sheets/fs297/en/ (2012). Accessed 15 Sept 2012.

2. Bertuccio P, Chatenoud L, Levi F, Praud D, Ferlay J, Negri E, Malvezzi M, La Vecchia C. Recent patterns in gastric cancer: a global overview. Int J Cancer. 2009;125:666-73. 
3. Australian Institute of Health and Welfare. Cancer in Australia: An overview, 2010. December 16, 2010. Available at: http:// www.aihw.gov.au/publication-detail/?id=6442472459 (2012). Accessed 10 Oct 2012.

4. Roder DM. The epidemiology of gastric cancer. Gastric Cancer. 2002;5 (suppl 1):5-11.

5. Theuer CP, Kurosaki T, Ziogas A, Butler J, Anton-Culver H. Asian patients with gastric carcinoma in the United States exhibit unique clinical features and superior overall and cancer specific survival rates. Cancer (Phila). 2000;89:1883-92.

6. Gill S, Shah A, Le N, Cook EF, Yoshida EM. Asian ethnicityrelated differences in gastric cancer presentation and outcome among patients treated at a Canadian cancer center. J Clin Oncol. 2003;21:2070-6.

7. Strong VE, Song KY, Park CH, Jacks LM, Gonen M, Shah M, Coit DG, Brennan MF. Comparison of gastric cancer survival following R0 resection in the United States and Korea using an internationally validated nomogram. Ann Surg. 2010;251:640-6.
8. Deurenberg P, Deurenberg-Yap M, Guricci S. Asians are different from Caucasians and from each other in their body mass index/body fat per cent relationship. Obes Rev. 2002;3:141-6.

9. Dhar DK, Kubota H, Tachibana M, Kotoh T, Tabara H, Masunaga $\mathrm{R}$, Kohno $\mathrm{H}$, Nagasue $\mathrm{N}$. Body mass index determines the success of lymph node dissection and predicts the outcome of gastric carcinoma patients. Oncology. 2000;59:18-23.

10. Inagawa S, Adachi S, Oda T, Kawamoto T, Koike N, Fukao K. Effect of fat volume on postoperative complications and survival rate after D2 dissection for gastric cancer. Gastric Cancer. 2000;3:141-4.

11. Kim J, Mailey B, Senthil M, Artinyan A, Sun CL, Bhatia S. Disparities in gastric cancer outcomes among Asian ethnicities in the USA. Ann Surg Oncol. 2009;16:2433-41.

12. Byfield SAD, Earle CC, Ayanian JZ, McCarthy EP. Treatment and outcomes of gastric cancer among United States-born and foreign-born Asians and Pacific Islanders. Cancer (Phila). 2009; 115:4595-605. 